\title{
El mandato pedagógico institucional: ¿Cuál perfil de ser humano educar?
}

The institutional pedagogical mandate: which human profile to educate?

\section{Volumen 17, Número 3 \\ Setiembre-Diciembre}

pp. 1-28

DOI: http://dx.doi.org/10.15517/aie.v17i3.29620

\author{
Victoria González García
}

Revista indizada en $\underline{\text { REDALYC, }}$ SCIELO

Revista distribuida en las bases de datos:

LATINDEX, DOAJ, REDIB, IRESIE, CLASE, DIALNET, SHERPA/ROMEO, QUALIS-CAPES, MIAR

Revista registrada en los directorios:

ULRICH'S, REDIE, RINACE, OEI, MAESTROTECA, PREAL, $\underline{\text { CLACSO }}$ 


\title{
El mandato pedagógico institucional: ¿Cuál perfil de ser humano educar?
}

The institutional pedagogical mandate: which human profile to educate?

\section{Victoria González García ${ }^{1}$}

\begin{abstract}
Resumen: En este ensayo se comparten hallazgos de la investigación "Relacionando Pedagogía, Currículo y Didáctica: el caso de las carreras de la Universidad de Costa Rica reacreditadas ante el SINAES". Esta investigación concibe la educación como un proceso de formación relativo a los seres humanos. Asimismo, elabora una propuesta con respecto a la descripción genérica de un perfil profesional del egresado de esta Universidad. Este perfil o mandato pedagógico institucional se constituye como una guía para las iniciativas de mejora curricular y didáctica en la institución, y fue construido a partir del trabajo con distintos docentes y los lineamientos existentes en documentos institucionales y en entrevistas a personas que coordinan esfuerzos relacionados con este tema. Al respecto, se parte del análisis de la misión universitaria considerada como la base de lo que hemos denominado "mandato pedagógico institucional". A partir de dicho mandato se realiza una reflexión sobre los orígenes de la Universidad de Costa Rica para identificar el contexto de su creación, la influencia en el modelo educativo costarricense y la pertinencia actual de dicha misión desde el Estatuto Orgánico de la Universidad. Con base en este ejercicio académico se construye el referido perfil con el fin de que guíe los distintos esfuerzos curriculares desarrollados en la Institución y en las distintas iniciativas de mejora docente. Se concluye que el perfil refleja el ideal universitario derivado del deseo o aspiración que orienta a esta Casa de Estudios Superiores con la razón de ser de la Universidad.
\end{abstract}

Palabras clave: pedagogía, educación superior, perfil de egreso, mandato pedagógico institucional

\begin{abstract}
This essay presents research findings "Linking Education, Curriculum and Teaching: The Case of the careers of the University of Costa Rica re-certified by SINAES". This research approaches the education as the training process that concerns to human beings. Also, it proposes an Institutional Pedagogic Mandate created with the generic description of a graduated human being profile from this university. This profile or Institutional Pedagogical Mandate, is proposed as a guide for the curricular and didactic improvement and it was built from the work of different professors, the existing guidelines in institutional documents and interviews with people who coordinate efforts related to this topic. Moreover, the "Institutional Pedagogic Mandate" is based on the mission of the university, as is denominated in the essay. Due to that mandate, there was done a consideration about the origin of the University of Costa Rica in order to identify the context of its creation, the influence of the educative Costa Rican model and the current pertinence of the mission from the organic statute of the University of Costa Rica. Based on that academic task it is created the profile to guide the different curricular efforts develop in the institution and another initiatives of professors training. It is concluded that the profile reflects the university's ideal from the de desire or aspiration which guides this High educational institution with the reason for being of the university.
\end{abstract}

Keywords: pedagogy, higher education, graduate profile, education institutional mandate

\footnotetext{
1 Docente Catedrática de la Universidad de Costa Rica. Investigadora, docente y asesora en pedagogía, diseño curricular e innovación docente en la Universidad de Costa Rica.
}

Dirección electrónica: victoria.gonzalez@ucr.ac.cr

Ensayo recibido: 18 de octubre, 2016

Enviado a corrección: 26 de abril, 2017

Aprobado: 5 de junio, 2017 


\section{Introducción}

Las instituciones educativas, como todas las organizaciones, son creadas con un fin, con una misión. En el caso de la Universidad de Costa Rica (UCR), desde sus orígenes, esta misión ha estado implícita en el Estatuto Orgánico, el cual hace mención al espíritu universitario, de cultura superior, creación, construcción de conocimiento, espíritu científico y otros. El presente ensayo plantea que la misión universitaria constituye un Mandato Pedagógico Institucional, que puede ser concretado mediante un perfil de egreso genérico.

En la presente reflexión se aborda, inicialmente, los orígenes de la UCR, para comprender, por una parte, el contexto en que fue creada, así como su influencia en el modelo educativo costarricense. Por otra parte, se revisó la presencia y validez de lo indicado en el Estatuto Orgánico, mediante la revisión de los distintos proyectos e instancias de la Vicerrectoría de Docencia y del Departamento de Docencia Universitaria (DEDUN) así como a través de distintas entrevistas a personas que dirigen estos proyectos e instancias y a profesores de distintas Unidades Académicas.

Este ejercicio permitió concretar el perfil de egreso genérico propuesto en este ensayo, con el fin de que guíe los distintos esfuerzos curriculares desarrollados en la Institución y en las distintas iniciativas de mejora docente.

\section{El mandato pedagógico universitario}

La Casa de Enseñanza de Santo Tomás (1814-1888) fue la primera institución estatal de Educación Superior en Costa Rica, se creó con el fin de contribuir con el desarrollo del país, al educar profesionales en ciencias básicas, ciencias de la salud, ingeniería, bellas artes y derecho. Dichas profesiones impulsaron el desarrollo costarricense a fines del siglo XIX e inicios del siglo XX. Esta Universidad, que funcionó durante 76 años, estaba dirigida, económica y políticamente, por clases dominantes bajo una visión oligarca². Después de ser cerrada esta Casa de Enseñanza, y de una pausa de 52 años, en 1940, se crea la UCR bajo principios que orientan al desarrollo de una institución universitaria como la conocemos hoy día, con diferencias cualitativas respecto a la Universidad de Santo Tomás, pues se sustentó en principios que le dieron sentido de movilidad social, y la concepción de la Educación Superior como un derecho para toda la población costarricense.

\footnotetext{
2 Se entiende oligarquía como la forma de gobierno en la cual el poder político es ejercido por un grupo minoritario que tiene el poder e influencia en un determinado sector social, económico y político (http://dle.rae.es/?id=R03HOdF).
} 
El 7 de marzo de 1941, la Universidad de Costa Rica abrió sus puertas en el céntrico barrio capitalino González Lahmann, con 719 estudiantes matriculados [...] Ya desde su comienzo era parte de su modelo organizativo la autonomía con la que hoy cuenta la universidad, soñada así por Luis Demetrio Tinoco, y se indicaba en el artículo 4 de su ley constitutiva que buscaba la autonomía de gobierno, administración y financiera. Desde un principio se propuso el estatus de la universidad como pública, por ser un servicio que garantiza la igualdad de oportunidad del acceso a la educación y que está ligado a la democracia. (Universidad de Costa Rica, 2015, párrs. 4-5)

El proyecto pedagógico de la Casa de Enseñanza de Santo Tomás respondía a la educación de un ser humano que ejerciera una profesión liberal y que, mediante el ejercicio de su profesión, influenciara los sectores social, económico y político costarricense. Esta educación era dirigida, como población meta, a la juventud costarricense perteneciente a la clase dominante. No obstante, la iniciativa de crear la UCR tuvo otro sentido, al ser creada para responder a la necesidad de contar con una institución estatal y autónoma ${ }^{3}$, así como con una institución de cultura general y humanística dirigida a todo el pueblo costarricense para formar un ser humano democrático ${ }^{4}$, con acceso a oportunidades de formación profesional, sin ningún tipo de discriminación, que le permitieran contribuir con la construcción y transformación de la sociedad costarricense cada vez más justa e inclusiva. Lo anterior implicó que el mandato educativo institucional fuese formar seres humanos críticos, propositivos, democráticos, conscientes de sus responsabilidades sociales y con valores como justicia social, respeto y solidaridad, entre otros. "En 1957 la Universidad se definió a sí misma como de cultura general humanística y su organización se da por las siguientes tres secciones: Humanidades (ciencias y letras), facultades 0 escuelas profesionales y la Facultad de Estudios Superiores" (Universidad de Costa Rica, 2015, párr. 8).

La UCR, desde sus inicios, influye en el pensamiento nacional y en la formulación de políticas en el campo educativo, pues cuenta con una persona que la representa en el

${ }^{3}$ Esta autonomía, en toda su dimensión, implica, por una parte, la capacidad de tener su propio gobierno, administración y presupuesto, y, por otra parte, que "la generación de conocimiento, la formación de alto nivel y la puesta del conocimiento al servicio de la sociedad -funciones sustantivas que definen el quehacer académico de la institución-; se lleven a cabo en un amplio espacio de ejercicio de la libertad de pensamiento, el diálogo, la crítica y la reflexión" (http://www.ucr.ac.cr/autonomia/). Este concepto es clave para definir el accionar de la Universidad, tanto académico como legal y administrativo.

${ }^{4}$ Se entiende democracia como la forma de gobierno cuyo poder político es ejercido por los ciudadanos, la soberanía reside en el pueblo y se respetan los derechos individuales, sin discriminación de etnia, sexo, religión (http://dle.rae.es/?id=C9NX1Wr). 
Consejo Superior de Educación. Por eso, cuando se redacta la Ley Fundamental de Educación, en el año 1956, cuyos representantes de la universidad eran los señores José Joaquín Trejos y Carlos Monge Alfaro, la Universidad, y el Consejo Universitario de ese entonces, tienen una participación activa. Los aportes de la UCR en la redacción del texto son muy importantes, especialmente en lo concerniente a "la división en ciclos de la enseñanza secundaria" (Consejo Universitario, 1956a, p.2), pues se discutía la pertinencia de configurar la educación secundaria con una orientación cristiano-occidental y de corte prevocacional, frente a una propuesta laica sin sesgos confesionales y valiosa en sí misma como proceso humano.

En este sentido, en el seno del Consejo Universitario, se hacen importantes aportes a la redacción de este proyecto de Ley. El tema de la educación secundaria es analizado en varias sesiones del Consejo Universitario, y para el 21 de mayo de 1956 se acuerda presentar ante el Consejo Superior de Educación las observaciones verbales y escritas para que "traten de incorporarlas a su dictamen, y si ello no fuere posible que las presenten al Consejo Superior de Educación como aportaciones individuales de los miembros del Consejo Universitario" (Consejo Universitario, 1956b, p. 13).

Una vez finalizada la redacción en el seno del Consejo Superior, el proyecto de Ley es presentado al presidente de Gobierno, sesión de trabajo en la que no se le hacen modificaciones por lo que ese texto fue presentado a la Asamblea Legislativa para el trámite correspondiente (Consejo Universitario, 1956b, p. 188).

El Proyecto de Ley Fundamental de Educación disfrutó de una amplia acogida en todos los sectores del país. Pocas críticas se hicieron a los principios y fines que le sirvieron de fundamento [...] todos los diputados se manifestaron desde un principio a favor del documento redactado por el Consejo Superior de Educación [...] pasó por unanimidad. (UCR, 1957, p. 189)

La afirmación anterior data del año1957, fecha en que se aprueba la Ley Fundamental de Educación. En ella se concibió la educación como un derecho de todo habitante de la República, y al Estado como responsable de garantizar ese derecho de la forma "más amplia y adecuada" aplicable a todos los niveles educativos, desde el preescolar hasta la educación superior o universitaria (Asamblea Legislativa, 1957, pp. 1-2). En dicha ley se precisan los fines de la educación costarricense, de los cuales puede inferirse el perfil de ser humano que se pretendía formar en el país, un ser humano que se caracterizara por ser amante de su Patria, consciente de sus deberes, de sus derechos y de sus libertades fundamentales, con 
sentido de responsabilidad y respeto por la dignidad humana, que viviera en democracia, solidaridad y cuidara su herencia cultural (Asamblea Legislativa, 1957, p. 1).

La Ley Fundamental de Educación legitimó a la UCR ${ }^{5}$, pues la definió así:

La Universidad de Costa Rica es una institución de cultura superior que goza de independencia en el desempeño de sus funciones y de plena capacidad jurídica para adquirir derechos y contraer obligaciones, así como para darse su organización y gobierno propios. (Asamblea Legislativa, 1957, p. 5)

Asimismo tenía la autoridad exclusiva de entregar títulos profesionales a partir de sus procesos de formación de profesionales en Costa Rica. Al respecto, Susan Francis (comunicación personal 8 junio 2016) expresa que “[...] en la Ley Fundamental de Educación, que es posteriori a la creación de UCR, la educación superior tiene finalidades asociadas a los fines de la educación costarricense".

A lo largo de sus 75 años, la UCR ha evolucionado hasta ser, hoy en día, una institución educativa que imparte 136 carreras en todo el país en sus 12 recintos universitarios (UCR, 2016), organizados en seis sedes regionales universitarias: Guanacaste, Puntarenas, Alajuela, Turrialba, Limón y Golfito y la Sede Interuniversitaria de Alajuela. Además, en el año 2001, fue declarada como Institución Benemérita de la Educación y la Cultura de Costa Rica, mediante el decreto № 8098.

En el año 1974 la Universidad decreta su Estatuto Orgánico, coherente con la Ley Fundamental de Educación, la cual da, a la Universidad, un carácter de institución de educación superior y cultura, autónoma constitucionalmente y democrática ${ }^{6}$, con la misión de dedicarse a la enseñanza, la investigación, la acción social, el estudio, la meditación, la creación artística y la difusión del conocimiento (artículo 1, Estatuto Orgánico); con el cometido de contribuir con las transformaciones que la sociedad necesita para el logro del bien común (artículo 4). Da una dirección concreta al proceso pedagógico de formación educativa, pues es espíritu de la Universidad graduar profesionales que contribuyan con la construcción de una mejor sociedad, gracias al desarrollo de actividades de vinculación con la sociedad, sea investigación, docencia o acción social.

\footnotetext{
${ }^{5}$ En ese momento en Costa Rica, como institución de educación superior, solo existía la UCR, por lo que la Ley solo menciona a esta institución. Actualmente esta Ley tiene alcance para todas las instituciones públicas de educación superior.

6 Las palabras en itálica, muestran aquellos aspectos que, a criterio de la investigadora, determinan el mandato pedagógico de la UCR y deberían estar presentes en todas sus propuestas curriculares y didácticas que en la Universidad se desarrollen.
} 
Así también, la Universidad ha sido un ejemplo de conciencia crítica pues participa activamente en aquellos movimientos sociales existentes en el país dirigidos a defender los pilares de justicia social; por ejemplo, en la defensa del Instituto Costarricense de Electricidad contra el Tratado de Libre Comercio con Estados Unidos, la minería a cielo abierto, y otros (UCR, 2015, párr. 16-19). Todo este accionar impacta el proceso de formación de la población estudiantil, la cual tiene la oportunidad de participar y formarse en estas actividades, complementar su formación académica y conocer el contexto costarricense en el cual se desarrollará profesionalmente. En el año 2015, la UCR ocupó el puesto 21 del Ranking de Universidades en América Latina y el puesto 481, en el ámbito mundial (UCR, 2015, párr. 16-19).

Cada diez años se realiza el Congreso Universitario, en aras de reflexionar acerca de su quehacer y definir el rumbo para los siguientes diez años. Como producto del III Congreso Universitario, en los años 70, la UCR se estructuró en cinco Vicerrectorías: Docencia (intercambio de saberes docente-estudiante), Investigación (construcción y producción de conocimiento), Acción Social (intercambio de saberes con la sociedad), Vida Estudiantil (acompañamiento de la población estudiantil) y Administración (apoyo administrativo).

Además del Estatuto Orgánico, la Universidad cuenta con las políticas universitarias y con el perfil de competencias genéricas para el personal docente. Este perfil determina los lineamientos que orientan a la población docente en el ejercicio de su labor, pues mediante la investigación construye conocimiento, mediante la docencia interactúa e intercambia saberes con la población estudiantil, y gracias a la acción social, lo hace con la sociedad en general. Todo lo anterior en un proceso de interacción formativa; es decir, en un "espacio de intercambio de ideas, emociones, pensamientos, intuiciones, argumentos y opiniones caracterizado por establecer relaciones horizontales, respetuosas, interesantes y desafiantes" (González, 2010, p. 61).

Es en ese espacio de interacción formativa que, en la Universidad, se han definido sus acciones sustantivas, es decir, las que le dan identidad, que la consolidan. Esta acciones son la docencia, la investigación y la acción social, y son consideradas por Flora Salas (comunicación personal, 20 abril, 2016) como parte de la estructura universitaria, y si no se realizan, la institución estaría "incompleta, pues el quehacer universitario se fundamenta en la interconexión de esas tres actividades; todas ellas realimentándose de manera permanente" (Flora Salas, comunicación personal, 20 de abril, 2016).

Existen esfuerzos más recientes para consolidar el quehacer académico de la 
Universidad, tales como las políticas universitarias aprobadas por el Consejo Universitario en el 2008 por primera vez, para los años 2010-2014 y, posteriormente, para los años 20162020. Estas políticas orientan "todas las actividades sustantivas de la Institución, se expresan mediante acciones concretas que fortalecen y mejoran el quehacer de la Universidad de Costa Rica" (Consejo Universitario, 2008, p. 1) y plantean que una docencia universitaria óptima es aquella que vincula la docencia con la investigación y la acción social, promueve procesos de autoevaluación, pertinencia, calidad y gestión de los planes de estudio, así como la formación, actualización y evaluación del talento humano de la institución, "según las necesidades y objetivos institucionales, buscando equilibrio y equidad entre las distintas áreas académicas" (Consejo Universitario, 2015, p. 2).

La Universidad también ha construido un perfil de competencias genéricas para el personal docente, aprobado en el año 2004, a partir de los desafíos y oportunidades presentes en la realidad latinoamericana con respecto a la educación superior (Consejo Universitario, 2004, p. 5). Este perfil concibe a la docencia de alta calidad como aquella que desarrolla actividades universitarias, tales como proyectos de docencia; investigación; acción social y gestión académico-administrativa, orientadas a enseñar, a aprender y a construir conocimiento, que promuevan el desarrollo integral del ser humano, la construcción de un pensamiento científico y de valores humanísticos del educando y la comunidad nacional, mediante un marco interdisciplinario de trabajo en equipo, responsabilidad compartida y de dominio de la especialidad, todo dentro de un compromiso con el desarrollo social (Consejo Universitario, 2004), por lo que organiza las competencias en las áreas de docencia, investigación, acción social, personal y ética-institucional. De estas políticas se desprende que la Universidad concibe la docencia, la investigación y la acción social según se lee a continuación:

A la docencia como el proceso planificado e intencionado, por el personal docente, en busca de la promoción de los aprendizajes significativos, la construcción de conocimientos, y la formación profesional integral del ser humano ... . La investigación como la constante elaboración de preguntas y la búsqueda de respuestas ... La Acción Social sería entonces interacción de saberes entre la universidad y la sociedad. ... Una docencia pertinente se enriquece con la investigación, construyendo nuevos conocimientos que pueden ser validados en comunidades mediante la acción social y la interacción con los participantes, en una relación compleja entre docencia, investigación y acción social. (González, 2012, pp. 11-12) 
Lo que está planteado en las líneas anteriores es que los esfuerzos institucionales deben orientarse hacia el desarrollo de procesos educativos para formar un ser humano con determinadas características, mediante procesos educativos que se vinculen con la investigación y la acción social. Es en este sentido que las distintas Vicerrectorías de la Universidad, y en particular la Vicerrectoría de Docencia, se encargan de supervisar el cumplimiento de este mandato pedagógico institucional.

La comunidad académica en general asume esta tarea liderada por la Vicerrectoría de Docencia (2016a), pues es la instancia que vela esencialmente por el cumplimiento de los lineamientos institucionales en materia pedagógica, curricular y didáctica. Asimismo, la Vicerrectoría de Docencia se encarga de aprobar los planes de estudio ${ }^{7}$, velar para que estos respondan a los desafíos que les plantea la realidad nacional, y respetar los principios y propósitos de la Universidad.

Esta Vicerrectoría también realiza investigación sobre métodos pedagógicos que orienten las estrategias didácticas; promueve la vinculación de los conocimientos universales con la realidad nacional mediante el fomento de la capacidad de análisis y crítica que le permita, a la población estudiantil, aplicar con creatividad los conocimientos. Además promueve la mejora continua del personal docente y realiza gestiones administrativas relativas al personal docente, como son permisos y licencias sabáticas, entre otros.

Por su parte, la Vicerrectoría de Investigación (2016) supervisa actividades académicas de construcción de conocimiento y contribuye de manera indirecta al logro de los objetivos pedagógicos de la Universidad, por medio de la promoción del desarrollo de investigaciones en independencia y autonomía para el desarrollo de Costa Rica, sin ningún tipo de subordinación a intereses extranjeros y vinculada a la docencia y a la investigación.

En cuanto a la Vicerrectoría de Acción Social (2016), esta lidera los esfuerzos de vinculación académica con la sociedad en el análisis y búsqueda de soluciones a las comunidades costarricenses, aportan en este proceso, los resultados de la investigación realizada mediante diferentes actividades como la Extensión Docente, la Extensión Cultural y el Trabajo Comunal Universitario. En el caso de la Vicerrectoría de Administración, sus funciones son básicamente dirigir, supervisar y evaluar la gestión administrativa con transparencia y eficiencia, y propiciar condiciones para la buena marcha administrativa de la Institución. Finalmente, la Vicerrectoría de Vida Estudiantil se encarga de liderar todos los

\footnotetext{
7 En adelante se llamará propuesta curricular, pues este término es más amplio que plan de estudios pues incluye los propósitos y referentes para la formación de las y los profesionales en respuesta a las necesidades de la sociedad costarricense.
} 
procesos de orientación y asistencia a la población estudiantil, de manera que se promueva la permanencia y el éxito académico (Consejo Universitario, 1974, Art. 50-54).

El ser una universidad pública, principio que hace una diferencia muy importante respecto de otras casas de estudio, la compromete con la sociedad costarricense para atender todas aquellas demandas relacionadas con la educación superior. Y tiene una característica particular, que no es común en todos los países del mundo, y es su autonomía. Esta autonomía le posibilita definir su forma de articulación de acciones de investigación, docencia y acción social y, a su vez, su vinculación con la sociedad y sus demandas sociales, tanto las actuales como las emergentes o las que eventualmente surjan. (Flora Salas, comunicación personal, 20 de abril, 2016).

Al asumir, la Vicerrectoría de Docencia, el liderazgo en la mejora del ejercicio docente, la Dra. Flora Salas, Directora de Políticas, Programas y Proyectos de la Vicerrectoría, quien dirige y coordina las iniciativas y proyectos, manifiesta que el mandato institucional está implícito en la misma universidad y que es tarea de cada docente revisarlo para orientar su quehacer.

El mandato de fondo está explicitado en el Estatuto Orgánico como gran marco de referencia. Tanto estudiantes como docentes, deberíamos conocer el Estatuto Orgánico, qué dice y qué manda, su fundamento, no solamente filosófico o sus funciones o propósitos, sino también su estructura, sus características y el desempeño de sus diferentes cargos. (Flora Salas, comunicación personal, 20 de abril, 2016)

Asimismo, en relación con la validez de ese mandato, Salas afirma que el Estatuto Orgánico todavía es válido, pues expresa una serie de "aspiraciones y que, como tales, continúan siendo un ideal por lograr", y se constituyen en el Mandato Pedagógico Institucional, planteado. Logro que depende, no solo de la universidad misma, sino también de la historia y la realidad de cada persona que integra la comunidad universitaria.

Yo pienso que la aspiración de que la universidad responda a las necesidades sociales, de ser consecuente, de perseguir la libertad de pensamiento, el desarrollo del conocimiento, de democratizarlo, de crear una comunidad académica en donde todas las personas que estamos dentro de la universidad formemos parte de ella y tengamos voz, no va a declinar. La Universidad de Costa Rica actual dista mucho de la de 1941, sin embargo, insisto, sus aspiraciones, que siguen siendo sus ideales, todavía no se logran en su totalidad. (Flora Salas, comunicación personal, 20 de abril, 2016) 
Salas prosigue afirmando que el mandato institucional "continúa teniendo vigencia. No le pondría ni quitaría nada", pues refleja la aspiración de una "universidad pública y completa. Esta aspiración sigue estando tan vigente hoy como el día uno". Es por esa razón que es importante revisar las instancias distintas que pertenecen a la Vicerrectoría de Docencia, pues todas ellas tienen impacto directo en la población docente. Estas son el Centro de Evaluación Académica, la Red Institucional de Formación y Evaluación Docente, el Programa de Cátedras Internacionales, la Unidad de Apoyo a la Docencia mediada por Tecnologías de la Información y la Comunicación, Docencia Multiversa y la Comisión de Proyectos de Docencia (Vicerrectoría de Docencia, 2016a, párr. 2).

El Centro de Evaluación Académica (en adelante CEA), dedicado a la evaluación e investigación curricular, tiene como propósito el "contribuir con el mejoramiento académico, mediante la investigación y evaluación de las diferentes dimensiones del fenómeno educativo propio de la Universidad de Costa Rica" (Centro de Evaluación Académica, 2016a, párr. 1), por lo que, entre sus actividades, realiza asesoría y colaboración estrecha, con la Vicerrectoría de Docencia, con la Rectoría, con el Consejo Universitario y con las distintas Unidades Académicas (Centro de Evaluación Académica, 2016a, párr.2). Entre las funciones relacionadas con este quehacer, y que están determinadas por el Reglamento del $\mathrm{CEA}^{8}$, se encuentran; por una parte, el quehacer curricular que implica la identificación, diagnóstico, evaluación e investigación del desarrollo curricular y la evaluación docente, y, por otra parte, el diseño de modelos de evaluación curricular. Adicionalmente, el CEA, como oficina técnicoadministrativa de la Vicerrectoría de Docencia, apoya la gestión y aseguramiento de la calidad de las carreras de la UCR a partir de la innovación y la evaluación en el desarrollo curricular y el desempeño docente con miras al mejoramiento académico, la certificación o la acreditación (Centro de Evaluación Académica, 2016b, párr. 1). Al respecto, su directora Marta Picado afirma:

El mandato institucional coloca la justicia social en el frente y, además, a la Universidad orientada hacia la sociedad, no hacia la sociedad en general, sino hacia sectores que demandan su actuar, como los sectores que requieren su apoyo o sectores que están generalmente desposeídos o que no tienen acceso a cierto servicio con el fin de que el conocimiento llegue a toda la población, sin ninguna clase de discriminación. Ese es el mandato que tiene en términos de solidaridad, en términos de justicia social, en términos de valores. Ese crecimiento integral de la persona, implica un crecimiento

\footnotetext{
${ }^{8}$ Puede consultar el documento en este enlace http://www.cu.ucr.ac.cr/normativ/centro evaluacion academica.pdf
} 
socioeconómico, también espiritual y luego social. El mandato institucional tiene más validez que nunca. (comunicación personal, 7 de setiembre, 2016)

La Red Institucional de Formación y Evaluación Docente (en adelante Rifed), busca la interrelación de las personas de la comunidad académica y potencia recursos con el fin de apoyar su quehacer docente, considerado "vivo, dinámico, descentralizado y deslocalizado" (Vicerrectoría de Docencia, 2016c, párr. 1). Rifed crea enlaces estratégicos entre personas e instancias, dentro y fuera de la Universidad y del país. Esta Red se expande a lo largo de toda la Universidad gracias a los programas de Bellas Artes, de la Facultad de Educación, de la Escuela de Ciencias Políticas, de la Escuela de Ciencias de la Computación e Informática, de Ciencias Básicas, de Sedes Regionales, del proyecto "Creciendo Juntos" de la Escuela de Administración de Negocios y "Universidad del Siglo XXI" de la Escuela de Estudios Generales. Como bien lo estipula la razón de ser de Rifed, todos estos programas tienen un común denominador: construir espacios dinámicos de interacción entre docentes para mejorar su acción pedagógica con sus estudiantes.

Rifed, que integra el Consejo Directivo de Docencia Multiversa, junto con la Unidad de Apoyo a la Docencia, mediada por Tecnologías de la Información y la Comunicación y el Centro de Informática, tiene como tarea fundamental:

Interconectar docentes en la UCR y apoyar redes de personas en la universidad, preocupadas por la docencia y por llevar innovación a la docencia. Una vez logrado esto, el mandato es ofrecer oportunidades de fortalecimiento como personas, como docentes y en redes, buscando la innovación. Se promueve actividades de mutua capacitación y se propicia el contacto con expertos nacionales e internacionales. (Eleonora Badilla, comunicación personal, 30 abril 2016)

Manifiesta Badilla que Rifed realiza lo anterior desde una perspectiva práctica, enfocada en la didáctica, con el propósito de que la población docente cuente con instrumentos innovadores para hacer docencia "desde el aprendizaje del estudiante, con base en un fundamento teórico-pedagógico", que promueva un cambio de enfoque, que de deje de mirar la enseñanza y que dé prioridad al aprendizaje, y, así, sustituir el vocabulario técnico, que, a su vez, es ajeno a muchos y muchas docentes. Con esto se trata de que los y las profesoras construyan un instrumental didáctico y tecnológico, en línea con esa pedagogía, es decir, que trabajan con una didáctica basada sobre todo en el aprendizaje, con una base constructivista. Para la Dra. Badilla, el mandato universitario está claro en el 
Estatuto Orgánico, pues le indica a la Universidad que debe apoyar la enseñanza y la transmisión de la cultura. Sin embargo:

En los últimos 40 o 50 años, ese mandato está quedando obsoleto porque actualmente la educación en general debe promover el aprendizaje más que la enseñanza, no solo por una cuestión conceptual y teórica, sino por una cuestión cultural y práctica: la transmisión de la información pues está en el aire, en la nube y en todos lados. (Eleonora Badilla, comunicación personal, 30 abril 2016)

Lo anterior implica que se transforma el rol de la población docente, pues deja de ser transmisora de información y experimenta "un rol mucho más interesante" dice Badilla. En ese contexto recomienda interpretar el Estatuto Orgánico, a través de la sustitución de la palabra enseñanza por aprendizaje, con lo cual se le da un sentido social a los procesos de aprendizaje, pues este se construye en la medida en que haya interacciones sociales, y en la actualidad no se vive ni culturalmente ni profesionalmente aislados.

La Universidad, continúa Badilla, tiene entre manos la formación de seres humanos que actuarán socialmente, y lo que orienta su quehacer es el Estatuto Orgánico. No obstante, es necesario tomar en cuenta que "cada disciplina tiene una imagen distinta", pues podría pensarse que: desde la administración se busca formar personas eficientes; desde las ciencias puras se buscan investigadores cuyo impacto social es indirecto; o desde las ciencias sociales, se espera profesionales que transformen la sociedad directamente. En este sentido, a su criterio, pueden observarse distintas visiones, entre ellas "la que más preocupa" que es aquella que parte de las demandas de pequeños sectores de la sociedad.

Un sector poderoso de la sociedad que quiere formar personas eficientes y con un gran énfasis en matemática y tecnología, una tecnología muy fría ¡verdad! Incluso es una gran demanda en el hemisferio occidental, lo que llaman en inglés STEM ${ }^{9}$ que es ciencia, tecnología, ingeniería y matemáticas. Entonces, vos ves que en muchos lados dice "desarrollamos el STEM"; esas son las disciplinas en las que los organismos internacionales están presionando a las universidades. Al respecto surge la propuesta del profesor del Medialab del Massachusetts Institute of Technology (MIT), llamado John Maeda, quien dice que no debe ser STEM, sino STEAM, refiriéndose a la incorporación del arte en esta fórmula, argumentando que la formación debe ser integral. (Eleonora Badilla, comunicación personal, 30 abril 2016)

9 Acrónimo en inglés cuyo significado es "Science, Technology, Engineering and Mathematics". 
La educación debe trascender la visión económica y ser considerada como un esfuerzo social para, mediante ella, ofrecer espacios de desarrollo al estudiantado, no solo en su área específica del saber disciplinar, sino también en otros aspectos como la creatividad, los valores, la intuición. Rifed ofrece al personal docente la oportunidad de fortalecer su conciencia pedagógica, por medio del reconocimiento de la importancia de brindar, al estudiantado, una formación integral que les lleve a conocer el mundo al que se enfrentarán. En este sentido, se estimula el contacto con otras disciplinas y un uso creativo de las tecnologías.

El Programa de Cátedras Internacionales, creado el segundo semestre del año 2013, pretende colocar, en la agenda universitaria y nacional, las relaciones entre Costa Rica y el mundo, estudiar su impacto histórico y su potencial en los diversos ámbitos de las sociedades latinoamericanas (Vicerrectoría de Docencia, 2016d, párr. 1). A la vez, impulsa "nuevas formas de internacionalización, tendientes a complementar los numerosos convenios de cooperación que permiten a la comunidad universitaria la actualización profesional y la colaboración con otras instancias fuera del país" (Vicerrectoría de Docencia, 2016d, párr. 2) y genera escenarios de punto de encuentro y diálogo. Son tres cátedras: Cátedra de Estudios de Corea y el Este Asiático, Cátedra de Estudios de África y el Caribe y Cátedra Wilhelm y Alexander von Humboldt en Humanidades y Ciencias Sociales, que están respaldadas por las Resoluciones VD-R-8969-2013, VD-R-8970-2013 y VD-R-9005-2013. Estas cátedras contribuyen con el logro del mandato pedagógico institucional, pues se constituyen en oportunidades de formación en cultura general. Estas cátedras no trabajan directamente con la población docente en el ámbito de la mejora del ejercicio pedagógico, sino que ofrece oportunidades de aprendizaje con respecto a los temas que estudia.

La Unidad de Apoyo a la Docencia mediada por Tecnologías de la Información y la Comunicación (en adelante Metics), "se ocupa del acompañamiento académico y soporte técnico al Portafolio Virtual de la Universidad de Costa Rica" (Vicerrectoría de Docencia, 2016b, párr. 1), apoya la docencia, la investigación y la acción social con ayuda de las Tecnologías de la Información y la Comunicación, ofrece servicios relativos a consultas curriculares, evaluativas y pedagógicas, formación docente mediada con tecnologías de la información y la comunicación (TIC), búsqueda de sitios, herramientas de producción multimedia, pizarra informativa y uso de aula virtual institucional, llamada MEDIACIÓN VIRTUAL (Vicerrectoría de Docencia, 2016b, párr. 3). Cuenta con un equipo interdisciplinario en las áreas de educación, comunicación, informática y diseño gráfico, que, con el fin de 
responder a las demandas de la población docente, mantienen vínculo permanente con el CEA, el Departamento de Docencia Universitaria (Dedun), el Centro de Informática, el Programa de Tecnologías Educativas Avanzadas (Protea) de la Facultad de Educación y el Sistema de Bibliotecas, Documentación e Información (Sibdi).

Por su parte, el proyecto Docencia Multiversa, que nace como una iniciativa de la Rectoría, vincula a Metics, Rifed y al Centro de Informática. Ofrece una docencia más flexible, inclusiva, transformadora social y profesionalmente, internacional, crítica y reflexiva, con lo cual se constituye en una "oportunidad de construir procesos de innovación y transformación de la acción docente y de los modelos curriculares en la educación superior" (Vicerrectoría de Docencia, 2016e, párr. 1). Su quehacer lo desarrolla mediante procesos formativos virtuales en materia de innovación docente y de transformación curricular. Su intención es poner en práctica modelos curriculares multiversos, en los cuales la población docente da sentido a sus actos educativos, y considera a la docencia como parte "inherente del acto educativo, por lo tanto inseparable del proceso de aprendizaje" (Vicerrectoría de Docencia, 2016 e, párr. 3). Esta propuesta contempla la presencialidad (presencia física en clases), la virtualidad (presencia física en un medio virtual) o los ambientes bimodales (mezcla de los dos anteriores), siendo posible trabajar desde distintos enfoques pedagógicos (Vicerrectoría de Docencia, 2016e, párrs. 6-7).

La Doctora Francis, directora de Metics, simultáneamente, coordinadora del Proyecto de Docencia Multiversa considera que el mandato institucional está vigente, pues:

Cuando se lee el Estatuto Orgánico, lo que se plantea es que la Universidad de Costa Rica tiene un papel fundamental en el desarrollo del país, de la sociedad costarricense, disminuyendo la desigualdad, la pobreza... . A modo general, se plantea también una sociedad democrática, justa, etc. [...] Esa misión se hace cada vez más necesaria y vigente. [...] Pienso que la universidad pensó perfilar un ciudadano comprometido con el desarrollo social. (Susan Francis, comunicación personal 8 junio 2016)

Para Francis, hace falta aclarar el "mecanismo de indagación" o "los mecanismos que operacionalizan esa misión”. Observación importante, pues la Universidad, al estar integrada por seres humanos que le dan vida y trabajan por el cumplimiento de esa misión, necesita que esas personas conozcan ese marco operativo de referencia que, en el caso pedagógico, es fundamental. El desafío se encuentra en que el mandato pedagógico institucional, referido a la formación de un ser humano, se ve influenciado por muchos factores que no 
necesariamente pueden ser evaluados por mecanismos operativos o cuantitativos. La Universidad, al contar con un mandato institucional, tiene, por una parte, la ventaja de ofrecer una guía, una orientación a toda su población y, por otra parte, el desafío de lograr su cumplimiento, tomando en consideración que quienes integran la comunidad universitaria son seres humanos diversos, con visiones de mundo distintas. Surge entonces la pregunta: ¿Cuáles estrategias son útiles para medir el cumplimiento de ese mandato institucional o marco de referencia? ¿Quiénes deberían participar en la evaluación del logro de ese mandato institucional o marco de referencia?

Es en ese sentido que la Dra. Francis enfatiza que habría que hacer cambios, como:

Crear y evaluar instrumentos para operacionalizar el mandato pedagógico institucional, para ver cuán consistentes son con la misión, para valorar, realmente, cuál es la interpretación que se está haciendo de esos conceptos, presentes en la misión, y si se cumplen.

Para ella, es importante tomar como punto de partida la misión establecida en el Estatuto, pues es ahí "donde está proyectada la misión de la universidad como una institución de apoyo y de detonante del desarrollo costarricense, tal y como se pensó inicialmente" (Susan Francis, comunicación personal 8 junio 2016).

En el caso concreto de Metics, y del proyecto Multiversa, responder al perfil de ser humano que la Universidad desea formar, es una tarea compleja, pues asumen ese mandato institucional con el afán de formar personas capaces de:

Desarrollar, cumplir, resolver problemas sociales, con actitud crítica, colaborativa e investigativa, con el uso de tecnologías de la información y la comunicación no como fin en si mismo, sino para potenciar las capacidades de investigación, de actitud crítica, de razonamiento crítico e inclusive colaborativo, sobre todo con MULTIVERSA, cuyo enfoque es de aprendizaje colaborativo. (Susan Francis, comunicación personal 8 junio 2016)

Es así que Metics y Multiversa pretenden evitar que el estudiante estudie solo, ensimismado o sin compartir o sin contribuir de manera integral a la sociedad, pues el mismo enfoque de "aprendizaje colaborativo lo pone, en sana teoría, en relación con las redes sociales y por lo tanto, lo pone en capacidad de identificar donde están las carencias" (Susan Francis, comunicación personal 8 junio 2016). En el transcurso de los diez años de existencia de Metics y los dos años de Multiversa, se ha dado énfasis en que la población docente trascienda el uso de las tecnologías como un fin en sí mismo y las asuma como un 
recurso más para apoyar el enfoque colaborativo en sus procesos de interacción con la población estudiantil. Este concepto y este esfuerzo son importantes, pues, tal y como afirma González:

Parece vislumbrarse una época en la que los seres humanos, para estar incluidos en las dinámicas sociales, necesitan ser capaces de analizar, recolectar, sintetizar información, elaborar hipótesis, imaginar respuestas y comunicarse. Sin embargo los mecanismos de comunicación están claramente impactados por la tecnología actual. ... Por lo tanto es necesario no solo contar con destrezas intelectuales para la comunicación, sino también con capacidad de utilizar la tecnología para comunicarse exitosamente. (2005, p. 06)

Metics y sobre todo, Multiversa impulsan la inclusión de las tecnologías en espacios de aprendizaje por parte del personal docente o investigador, cuya conciencia social y actitud crítica les motive a hacerlo. Metics ha desarrollado esfuerzos para que la población docente no mire la tecnología como un recurso con el que ha tenido que luchar y lidiar, sino más bien que la vean como un recurso accesible, fácil de usar y que mejora sus procesos investigativos y didácticos.

La Comisión de Proyectos de Docencia es una instancia de la Vicerrectoría de Docencia, respaldada por la Resolución VD-R-9155-2014, que ofrece a la población docente la oportunidad de reflexionar sobre su quehacer docente y mejorar los procesos de enseñanza aprendizaje mediante la formulación de proyectos docentes inscritos en el Sistema Institucional existente al efecto. "De la misma manera, se brinda un espacio para explorar diferentes enfoques de enseñanza y aprendizaje y los nuevos papeles docentes, más acordes con las nuevas circunstancias culturales, y con las características del estudiantado universitario actual" (Vicerrectoría de Docencia, 2016f, párr. 2).

Estos proyectos, formulados por docentes de la Universidad, están dirigidos al mejoramiento de habilidades pedagógicas del personal docente, y estructurado a partir de algunos de los siguientes ejes: reflexión-acción sobre la práctica pedagógica; calidad de la docencia; adquisición de herramientas teóricas, conceptuales y metodológicas para el ejercicio docente; desarrollo integral del personal docente y de la población estudiantil; mejora de los procesos de enseñanza-aprendizaje; generación de recursos didácticos; utilización de tecnologías de la información y comunicación; promoción de evaluación alternativa y reflexión sobre las nuevas competencias docentes y estudiantiles (Vicerrectoría 
de Docencia, 2016f, párr. 4).

La Comisión, por lo tanto, desarrolla acciones en dos vertientes. Por una parte, en el desarrollo de instrumentos para las Comisiones de Docencia de las distintas Unidades Académicas. Por otra parte, la revisión, aprobación y seguimiento de proyectos de docencia, inscritos por docentes de las distintas Unidades Académicas. Con respecto al mandato pedagógico institucional, en la Comisión de Proyectos de Docencia se considera que:

Lo que dicta el Estatuto Orgánico de la Universidad. Y uno pensaría, si me voy a trabajar a otra universidad, ¿Cuál sería la diferencia con la UCR? Hay una responsabilidad y un cierto prestigio al trabajar aquí que marca un sentido de calidad y de aprovechamiento de los recursos importante. Luego, es la Universidad de Costa Rica, ubicada en un país específico en un momento dado; entonces es una Universidad qué debería estar constantemente mirando que es lo que está pasando en la sociedad y adaptarse a eso. (Elsiana Guido, comunicación personal, 23 agosto 2016)

Otro espacio universitario existente con el propósito de mejorar la docencia es el Departamento de Docencia Universitaria (Dedun), que pertenece a la Escuela de Formación Docente de la Facultad de Educación. El propósito del Dedun se centra en el desarrollo de actividades académicas de docencia, investigación y acción social (Escuela de Formación Docente, 2016a, párr. 1) y desarrolla varias iniciativas, a saber: el curso de Didáctica Universitaria que aborda aspectos teóricos para el mejoramiento de la labor docente y se dirige a la población docente; el Programa Docente, constituido por la Licenciatura en Docencia Universitaria, la Actualización Docente (cursos de corta duración), la Maestría en Docencia Universitaria y los Talleres de Asesoría Pedagógica. También, desarrolla investigación y acción social en sus áreas de trabajo, tales como la pedagogía universitaria y las tecnologías de información. También desarrollan materiales y textos para la Educación Superior (Escuela de Formación Docente, 2016b, párr. 1-10).

Para Patricia Marín, directora del Dedun, la mejora continua en la didáctica de la educación superior, por parte del personal docente universitario, se da en el marco de procesos de acreditación de las carreras en las cuales imparten lecciones, por lo que se hace necesario trascender este aspecto y motivar la mejora continua en didáctica de la educación superior con la motivación de promover mejores procesos de aprendizaje en la población estudiantil que atienden. A este respecto, y en respuesta al mandato pedagógico institucional, manifiesta que "deben analizarse todos los componentes o los principios emanados de la misión de la Universidad, tanto desde lo externo como de lo interno, 
orientado al trabajo que realiza la Universidad y en el contexto que nos encontremos" (Patricia Marín, comunicación personal, 24 de junio, 2016). De tal manera que estos componentes orienten las acciones docentes universitarias. Patricia Marín también argumenta que:

Es importante contextualizar dicho mandato universitario pues, como toda obra política, se inscribe en un contexto histórico, por lo que debe ser revisado constantemente. Somos una institución de educación superior y cultura, pero más allá, la Universidad tiene una responsabilidad social muy grande, y debería valorarse si la misión engloba todo, los mandatos o las exigencias o las solicitudes que la sociedad le está haciendo actualmente. Para mí mantiene validez, lo que habría que revisar es si estipula todo lo que en realidad hacemos en la universidad. (comunicación personal, 24 de junio, 2016)

Como pudo notarse, todas las personas entrevistadas coinciden en que el Mandato Pedagógico Institucional, estipulado en el Estatuto Orgánico, mantiene su validez y requiere revisión constante en función de las necesidades y demandas del contexto en que se encuentre la institución, sin olvidar el marco de autonomía universitaria y, sobre todo, la misión social con la que fue creada la universidad pública.

\section{Importancia de la redacción del Mandato Pedagógico Institucional en formato de perfil genérico de egreso de la UCR}

El ejercicio de construir un perfil de ser humano que se constituya en la guía para cumplir el Mandato Pedagógico Institucional, es —o debería ser - dinámico pues siempre estará determinado por la misión de la institución educativa y por la sociedad en la cual se encuentra. El propósito de este trabajo es plantear que el Mandato Pedagógico Institucional puede estar redactado en términos de un perfil de egreso genérico, tal y como se manifiesta a continuación:

El perfil de egreso es, junto con el plan y los programas de estudio, la expresión materializada de una propuesta educativa para la formación de un profesional, en definitiva, es la orientación que se le da a la formación del profesional, expresada en el perfil de egreso. (Ysunza, 2010, p. 35)

Por lo que al hacer referencia al concepto de perfil de egreso, se habla de un proceso educativo, es decir de una serie de actos educativos. 
Los actos educativos son aquellos actos mediante los cuales se forman o educan los seres humanos, es decir, todo acto educativo nos lleva a la formación de determinado tipo de ser humano. Los actos pedagógicos son aquellos que se desarrollan con una intencionalidad dirigida al aprendizaje en espacios didácticamente pensados. La pedagogía, entonces, es la ciencia que estudia esos actos educativos y que, por lo tanto, construye teoría educativa que nos orienta en el diseño de actos pedagógicos.

En otras palabras, la expresión "Mandato Pedagógico Institucional" expresa el compromiso que toda institución educativa asume ante su población meta, sea cual sea el nivel educativo, desde el inicial al superior, y se traduce en la formación de un determinado perfil de ser humano definido, explícito y coherente con las ideas inspiradoras que la crearon. Y, a partir de él, es posible determinar el enfoque pedagógico y sus respectivas estrategias institucionales, curriculares y didácticas.

"La pedagogía es una ciencia porque tiene un objeto de estudio (la educación), un método de estudio (interpretativismo) y una producción teórica" (González, 2014, p. 55). Es la pedagogía la que nos ofrece el sustento teórico y las premisas necesarias para dirigir los esfuerzos en pro de formar personas, específicamente en el campo curricular, didáctico y de mejora docente. La pedagogía "piensa en el proceso educativo del ser humano. Esto significa que estudia todas las manifestaciones sociales en las que se intercambia y construye conocimiento" (González, 2014, p. 56).

La Universidad, como institución educativa y de cultura superior, necesita cumplir con este requisito y orientar su propósito pedagógico, es decir, la formación de profesionales en distintos campos hacia la comprensión y transformación del mundo y transcender la visión instruccionista o técnica que ha prevalecido desde años atrás. Tal y como lo afirma Flora Salas (comunicación personal, 20 de abril, 2016):

Desde sus inicios, la universidad se interesa por formar gente que sepa hacer "algo" con cierto grado de complejidad y con cierto nivel de formación y conocimiento especializado, de tipo científico-técnico, es decir por formar profesionales en campos para el desarrollo de la ciencia en el nivel teórico. En la Universidad de Costa Rica, en particular y con la influencia de la Revolución Industrial del Siglo XVIII y de la universidad francesa, se trabaja en pro de la formación profesional con aprendizajes científicos y disciplinares así como con otros de cultura general, o lo que se ha llamado en el desarrollo del currículum "el perfil académico" y "el perfil profesional"; esas dos cosas se tienen que sumar para darnos como resultado final a una persona profesional 
que sea, no solo muy buena en el nivel disciplinar y el desempeño en su campo, sino que sea también una persona responsable socialmente y que sea capaz de ver en su práctica profesional una ética de fondo, no solo en la práctica profesional, sino de compromiso con la sociedad. (Flora Salas, comunicación personal, 20 de abril, 2016)

La UCR, desde que inició, incluyó en su visión pedagógica la formación en cultura general y académica, asumiendo que la educación superior no es únicamente la transmisión de información ni la formación exclusivamente técnica, sino también lo es la oferta de oportunidades para el desarrollo de sus "capacidades para entender el mundo, la propia vida así como la toma de decisiones" (Flora Salas, comunicación personal, 20 de abril, 2016). En este sentido, la UCR, como institución educativa, que desarrolla procesos pedagógicos, tiene la finalidad de que las personas que se forman en ella puedan participar del mundo, realizar transformaciones, tener conciencia sobre sus decisiones. En palabras de Salas, "una formación integral, humanista, una formación realmente universitaria", en donde se aprende la disciplina y otras áreas como la filosofía, arte, literatura, comunicación y "todo esto que en el fondo se llama la alta cultura o la cultura más relevante para el acervo de conocimientos y valores de la humanidad" (Flora Salas, comunicación personal, 20 de abril, 2016).

En esa misma línea, Susan Francis (comunicación personal 8 junio 2016) sugiere crear o revisar los instrumentos dirigidos a evaluar el logro de la misión institucional en un esfuerzo por interpretar o conocer cómo se está interpretando esa misión, "para saber si realmente se está cumpliendo con construir esa idea de sociedad" con la que se compromete la Universidad y, en un ámbito más específico, mejorar la gestión de currículo, superar el hecho de contratar docentes y administrarlos, generar espacios de mejora del personal docente en relación con los procesos de formación de la población estudiantil hacia el logro del perfil de la profesión o la disciplina que imparten.

Tanto para Patricia Marín (comunicación personal, 24 de junio, 2016), como para Eleonora Badilla (comunicación personal, 30 abril 2016) y para Elsiana Guido (comunicación personal, 23 agosto 2016), la misión y la visión de la Universidad contribuyen aportando "el ideal" de perfil humano a formar. Para Marín, además, "el énfasis debe estar en la formación con responsabilidad social, de manera explícita en los procesos formativos, incluyendo "valores éticos y morales"; agrega Badilla que "el movimiento estudiantil es corresponsable de hacer realidad esta intención”, y complementa Elsiana Guido (comunicación personal, 23 agosto 2016), que "el egresado de la Universidad debería distinguirse positivamente del 
resto de las universidades. No debería estar implicado en actos de corrupción contra el mismo Estado que le financió su educación".

Ahora bien, definir el tipo de ser humano con el cual la Universidad se compromete a educar de acuerdo con el mandato pedagógico institucional, puede ser caracterizado en función de sus valores, actitudes, habilidades, destrezas y conocimientos. Esto es clave para el logro de varias tareas. Una es que, en respuesta a ese perfil de ser humano, puede determinarse el enfoque o enfoques pedagógicos que orienten ese proceso de formación. Otra, es que se garantice contar con un marco de referencia para orientar todas las iniciativas académicas, llámense proyectos de docencia, investigación o acción social, dirigidos a la formación de este ser humano. Una tercera es que, a partir de lo anterior, puedan determinarse enfoques curriculares y didácticos que guíen tanto las propuestas curriculares como las opciones metodológicas de los cursos que se imparten en la Universidad. Y, finalmente, pueden también orientarse los esfuerzos de evaluación, es decir, conocer si se logra efectivamente formar ese ser humano imaginado. La UCR puede concentrar sus esfuerzos en realizar estas tareas para lograr su Mandato Pedagógico Institucional.

Sería óptimo definir un perfil de ser humano únicamente a partir de características observables. Sin embargo, conscientes de que el aprendizaje es un proceso interno que no necesariamente es evidente a los ojos de los demás, el desafío es mayor, pues será necesario describir a ese ser humano más allá de conductas observables. Una vez lograda esta descripción pueden mirarse las oportunidades curriculares y didácticas en los cursos de formación humana y profesional pues “... la vivencia de la docencia y de la enseñanza y del aprendizaje ocurre en las aulas" (Flora Salas, comunicación personal, 20 de abril, 2016). Finalmente es en esos espacios donde se concreta la formación educativa.

Al redactar un perfil profesional, aconseja Flora Salas (comunicación personal, 20 de abril, 2016), es importante tomar en cuenta tres cuidados especiales. Uno, evitar la redundancia de conceptos, otro, evitar el uso de conceptos metafísicos que cuesta medir o evaluar y, el último, evitar el lenguaje ambiguo. La tarea clave, y en este aspecto coinciden Salas, Francis y Picado, es responder ¿cómo medir el logro?, ¿cómo evaluarlo?, ¿qué instrumentos son necesarios para evaluar el cumplimiento del perfil? ¿cómo realizar una medición organizada para evaluar el cumplimiento de este perfil? (Flora Salas, comunicación personal, 20 de abril, 2016; Susan Francis, comunicación personal 8 junio 2016; Marta Picado, comunicación personal, 7 de setiembre, 2016). Patricia Marín (comunicación 
personal, 24 de junio, 2016) sugiere que, para fortalecer el perfil de ser humano construido, se tome en cuenta la opinión de los empleadores, quienes opinan que:

Los profesionales que salen de la universidad están muy bien preparados en su disciplina conceptualmente, o sea tienen una buena formación en el ámbito conceptual, pero digamos, son personas que tienen muy poca formación en lo que se llama trabajo en equipo, toma de decisiones bajo imprevistos o bajo situaciones de riesgo, o situaciones imprevistas. (Conare, 2007, citado por Patricia Marín, comunicación personal, 24 de junio, 2016)

Para Marta Picado (comunicación personal, 7 de setiembre, 2016) es indispensable que se incluya en ese perfil "la curiosidad en investigación, la ética en la que quepamos todos y todas, así como la integración teórico-práctica. No hay práctica sin teoría, ni teoría sin práctica. El estudiante generalmente se queda a nivel del texto y no ve el contexto".

Susan Francis (comunicación personal 8 junio 2016) recomienda hacer énfasis en la formación humana, no individual, sino colectiva, de manera que "seamos conscientes de cuál diferencia hará que el perfil esté definido en términos de comunidad y no en términos de individuos", y adicionalmente, visualiza el aspecto inclusivo. Aspectos que deben ser tomados en consideración e incluidos en cualquier perfil de ser humano propuesto pues, el aprendizaje es un proceso social, el cual se produce en interacción con las otras personas y con el contexto en general y no existe una sociedad justa socialmente sin su inclusión.

Este trabajo aporta un perfil genérico de ser humano para educar en la UCR, construido gracias a la revisión documental, a los aportes de las personas entrevistadas y a espacios de reflexión con algunos y algunas docentes de la institución, integrantes de cuatro Unidades Académicas; a saber: las Escuela de Administración Pública, de Enfermería, de Psicología y la Facultad de Microbiología, Unidades Académicas participantes en la investigación "Relacionando Pedagogía, Curriculum y Didáctica: el caso de las carreras de la UCR reacreditadas ante el Sinaes en los años 2010-2014". La idea es que este perfil propuesto oriente los esfuerzos curriculares y didácticos que realice la Universidad en la formación educativa de profesionales de distintas disciplinas.

Luego de la reflexión compartida en líneas anteriores, se asume que el ser humano que la Universidad espera formar, en su proceso educativo, sea una persona capaz de construir conocimientos científicos y técnicos de su profesión, y relativos a su ejercicio laboral, a la historia de la humanidad y a su cultura, así como poder construir relaciones interpersonales establecidas en el marco de su disciplina. Esta persona también debe 
desarrollar habilidades de pensamiento crítico, curiosidad intelectual, auto-aprendizaje, diálogo, trabajo colaborativo y proactivo hacia la transformación y justicia social y vivir valores como el compromiso ambiental, solidaridad, paz, soberanía, disfrute del trabajo, humanismo, justicia, transparencia, respeto, democracia, responsabilidad, vivencia en diversidad y estética.

Ahora bien, la teoría curricular mexicana, que ha influenciado a la utilizada en la UCR, plantea que el perfil de egreso o perfil profesional refleja las "intencionalidades o estados meta terminales" (Díaz Barriga, citado por Ysunza, 2010, p. 53) y debe

Conjuntar una visión humanista, científica y social de manera integrada, alrededor de conocimientos, habilidades, destrezas, actitudes, valores, etcétera [así como] la delimitación de las áreas o sectores donde se llevará a cabo la labor del egresado, los principales ámbitos de la actividad profesional a realizar y las poblaciones 0 beneficiarios de su labor profesional. (Díaz Barriga, citado por Ysunza, 2010, p. 53)

Los perfiles de egreso de las carreras universitarias, en la UCR, responden a la política curricular del CEA y son definidos en función de los conocimientos, las habilidades/destrezas y las actitudes/valores (Centro de Evaluación Académica, 2015) por desarrollar durante el aprendizaje de la profesión, esto coincide con lo propuesto por la Universidad Autónoma de México, cuya política establece que el perfil de la persona egresada debe estar compuesto por "los conocimientos teóricos, metodológicos y técnicos que deberá poseer; las habilidades profesionales y los aspectos personales vinculados con actitudes y cualidades personales y profesionales" (Ysunza, 2010, p. 54).

Además, el perfil de egreso es básico en la toma de decisiones curriculares, tanto en aspectos estructurales como de contenidos del plan de estudios, pues expresa "de manera sintética y con un mayor nivel de precisión las directrices principales de la propuesta educativa[ ...]" (Ysunza, 2010, p. 54); con lo cual, el hecho de que la UCR cuente con un perfil genérico que guíe la construcción de los distintos perfiles de salida de las carreras que imparte, garantiza, o al menos promueve, que se cumpla con el Mandato Pedagógico Institucional. En otras palabras, un perfil genérico de la persona egresada de la UCR puede ser un instrumento de apoyo a las distintas Unidades Académicas en su tarea de cumplir con el Mandato Pedagógico Institucional. 


\section{Propuesta de Mandato Pedagógico Institucional o perfil genérico de egreso de la UCR}

Considerando todo lo anterior, se propone, a continuación, una propuesta de perfil de ser humano graduado de la universidad como instrumento para revisar los distintos perfiles profesionales de salida, apoyar su puesta en práctica y su respectiva evaluación.

La tabla 1 muestra un perfil profesional de egreso genérico y se ofrece a la comunidad universitaria como una guía para el diseño, revisión, actualización y modificación de las respectivas propuestas curriculares existentes y por crear en el seno de la UCR. Este perfil, estructurado en tres columnas, está inspirado en las reflexiones anteriores y refleja conocimientos genéricos, habilidades — destrezas y actitudes— valores, que servirán para la reflexión en el seno de cada Unidad Académica. No obstante, se propone, a continuación, una aproximación conceptual como aporte para la reflexión y el posible consenso.

Toda persona profesional necesita conocer la historia de su profesión, el contexto en el que se desarrolla así como las bases científicas de su quehacer profesional. También requiere conocer acerca del instrumental y técnicas específicas de la profesión para poder construir conceptos científico-profesionales que le orienten en la toma de decisiones y en la solución de problemas y desafíos sociales en el ámbito de la profesión. Es con base en esta dimensión del conocer que se construye la columna "conocimientos".

Además de lo anterior, se espera que las personas profesionales sean capaces de realizar determinadas tareas, de forma autónoma, correcta y en interacción con las personas - profesionales o no - con que le corresponda ejercer, por eso se habla de habilidades y destrezas, pues contempla "cada una de las cosas que una persona ejecuta con gracia y destreza" (http://dle.rae.es/srv/search?w=habilidad).

Las actitudes pueden ser comprendidas como la "disposición de ánimo manifestada de algún modo" (http://dle.rae.es/srv/search?m=30\&w=actitud), y los valores, como los principios que orientan la toma de decisiones en todas las dimensiones de la vida personal y profesional. De esta manera, con las actitudes y valores asumidos, la persona profesional que egresa de la UCR se caracteriza por una postura ética en pro del bienestar inclusivo, con compromiso con la justicia social y la consciencia crítica, tal y como lo indica el Estatuto Orgánico. 
Tabla 1. Propuesta de Mandato Pedagógico Institucional o perfil de egreso profesional de la UCR

\section{CONOCIMIENTOS \\ HABILIDADES/DESTREZAS}

Ejercicio profesional:

- Auto-aprendizaje

- Aprendizaje continuo

- Ciencias básicas o sociales según sea la disciplina

- Conceptos científico-teóricos que sustentan la profesión

- Técnicas, instrumentos y tecnologías específicas de la profesión

- Relaciones interpersonales en el marco de la disciplina

Humanidad/Sociedad:

- Diálogo

- Trabajo colaborativo

- Propositivo

- Análisis de teoría y contexto

- Manejo del tiempo

- Construcción de conocimientos

- Liderazgo

- Sana relación con población que se atiende

Humanidad/Sociedad:

- Historia de la Humanidad

- Cultura nacional y regional: artes, deporte, realidad nacional.

- Movimientos Políticos

Pensamiento crítico:

- Pensamiento crítico

- Conceptos y metodologías de investigación

- Conceptos y metodologías para desarrollo de proyectos

- Población que atiende en el marco de la profesión

- Comunicación escrita y oral de sus conocimientos. materna.

Pensamiento Crítico: sociales relativos a la equidad y la investigación.

- Participación en proyectos de transformación social

- Cuestionamiento, habilidades para hacer preguntas "reflexivas".
- Dominio de otro idioma y de su lengua

- Determinación de dilemas y problemas injusticia social, mediante procesos de

- Práctica laboral reflexiva e innovadora.

- Solución de situaciones especiales en el contexto de la profesión.

- Evaluación de impacto

- Capacidad de trabajo con otras profesiones (multi, inter y transdisciplinar)

Salud:

- Cuidado intrapersonal (autorregulaciónautocuidado)

- Cuidado interpersonal (interacciones y entornos saludables)

Fuente: Elaboración propia con base en la construcción colectiva entre la autora del presente ensayo, personas entrevistadas y diferentes docentes de las Unidades Académicas: Administración Pública, Enfermería, Microbiología y Psicología.
Comportamiento: rigurosidad intelectual, transparencia, soberanía, responsabilidad

Compromiso: con la paz, el buen trato al planeta y con sus interacciones, con la solidaridad, justicia.

Respeto:

a la diversidad de expresiones humanas, a la vida en democracia, al género, la inclusividad (discapacidad, diferencias étnicas, otros), la libertad, equidad, bioética.

\section{Estética:} disfrute del trabajo, creatividad e innovación, autocuidado 


\section{Reflexiones finales}

La idea más importante que se ha querido plasmar en este trabajo es que la UCR tiene un Mandato Pedagógico Institucional claramente definido y reconocido por la comunidad universitaria y manifestado por quienes coordinan iniciativas y proyectos dirigidos al ejercicio pedagógico en la Universidad. Este mandato no es una expresión simple, pues recoge el espíritu de creación de una universidad pública y costeada por el Estado Costarricense, reflejado en la Ley Fundamental de Educación y en el Estatuto Orgánico de la Institución. Este mandato, en buena teoría, debe dirigir, orientar y justificar todos los esfuerzos que realiza la UCR, tanto en docencia como en investigación y acción social.

El Mandato Pedagógico Institucional refleja aquella instrucción, ideal, deseo, aspiración con que fue creada la institución educativa, en este caso la Universidad, por lo que es irrenunciable y debería ser tomado en cuenta en todos los esfuerzos de construcción curricular y didáctica que se emprendan en el seno de este centro educativo, particularmente en docencia. No obstante, también debería orientar la investigación y la acción social. Pese a ello, el ámbito docente lleva la mayor responsabilidad, pues es en ese espacio donde se colocan todos aquellos conocimientos construidos mediante la investigación y se proyectan intercambios de conocimiento con la sociedad a través de la acción social.

Todas las personas que aportaron para la construcción del Mandato Pedagógico Institucional coinciden en que los distintos documentos orientadores de la Universidad son válidos, sobre todo lo indicado en el Estatuto Orgánico, y aclaran algunos aspectos fundamentales como: que este mandato continúa siendo una aspiración de la Universidad, que es importante que la Universidad analice la pertinencia de transformar su enfoque pedagógico, que mire más los procesos de aprendizaje que los de enseñanza y que incluya el uso de las tecnologías como apoyo de estos procesos. Asimismo dichas personas señalan que deben crearse estrategias e instrumentos para evaluar el logro de este mandato.

El hecho de proponer un perfil de ser humano clasificado en tres apartados (conocimientos, habilidades y destrezas, actitudes y valores) se realiza únicamente con fines instrumentales. La tarea esencial es cuidar que se dé una estrecha vinculación de estos tres, mediante estrategias curriculares y didácticas u otras que defina la Unidad Académica. 


\section{Referencias}

Asamblea Legislativa, Costa Rica. (1957). Ley Fundamental de Educación. San José, Costa Rica: Imprenta Nacional.

Centro de Evaluación Académica, Universidad de Costa Rica. (2016a). Centro de Evaluación Académica. San José, Costa Rica. Recuperado de http://www.cea.ucr.ac.cr/index.php

Centro de Evaluación Académica, Universidad de Costa Rica. (2016b). Quienes somos. San José, Costa Rica. Recuperado de http://www.cea.ucr.ac.cr/index.php/quienes-somos

Centro de Evaluación Académica, Universidad de Costa Rica. (2015). Diseño Curricular Universitario. Orientación para los procesos de diseño curricular. Recuperado de http://www.cea.ucr.ac.cr/media/diea/publicaciones/orientacion-diseno-curricular.pdf

Consejo Universitario, Universidad de Costa Rica. (2015). Políticas de la Universidad de Costa Rica 2016-2020 "Excelencia e Innovación con Transparencia y Equidad". La Gaceta Universitaria N 13-2015. San José, Costa Rica: Universidad de Costa Rica.

Consejo Universitario, Universidad de Costa Rica. (2008). Políticas de la Universidad de Costa Rica para los años 2010-2014. La Gaceta Universitaria N 40-2008. San José, Costa Rica: Universidad de Costa Rica.

Consejo Universitario, Universidad de Costa Rica. (2004). Perfil de competencias para el profesorado de la Universidad de Costa Rica. Gaceta Universitaria $N^{\circ}$ 39-2004. San José-Costa Rica: Universidad de Costa Rica.

Consejo Universitario, Universidad de Costa Rica. (1974). Estatuto Orgánico de la Universidad de Costa Rica. Recuperado de http://www.cu.ucr.ac.cr/normativ/estatuto organico.pdf

Consejo Universitario, Universidad de Costa Rica. (1956b). Acta de la Sesión № 822 celebrada el 21 de mayo de 1956. Universidad de Costa Rica. San José. Recuperado de http://www.cu.ucr.ac.cr/actas/1956/822.pdf

Consejo Universitario, Universidad de Costa Rica. (1956a). Acta de la Sesión $N^{\circ} 818$ celebrada el 02 de mayo de 1956. Recuperado de http://www.cu.ucr.ac.cr/actas/1956/818.pdf

Escuela de Formación Docente, Universidad de Costa Rica. (2016a). Departamento de Docencia Universitaria. Recuperado de http://docenciauniversitaria.ucr.ac.cr/

Escuela de Formación Docente, Universidad de Costa Rica. (2016b). Departamento de Docencia Universitaria: Actividades y servicios. Recuperado de http://docenciauniversitaria.ucr.ac.cr/index.php/inicio/actividades-servicios

González, Victoria. (2014). Innovar en docencia universitaria: algunos enfoques pedagógicos. Intersedes - Revista Electrónica de las Sedes Regionales de la Universidad de Costa Rica, 15(31), http://www.intersedes.ucr.ac.cr/ojs/index.php/intersedes/article/view/423/405

50-68. 
González, Victoria. (2012). La Innovación Docente: Reflexiones a Partir del Enfoque Histórico Cultural. Actualidades Investigativas en Educación, 12(1), 1-31.

González, Victoria. (2010). Interpretando el pensamiento complejo: un acercamiento a Lev S. Vygotsky. Revista Posgrado y Sociedad, 10(1), 38-63.

González, Victoria. (2005). Tecnología digital: reflexiones pedagógicas y socioculturales. Actualidades Investigativas en Educación, 5(1) 1-24.

Universidad de Costa Rica (UCR). (1957). Anales de la Universidad de Costa Rica 1957 [Versión digital]. Recuperado de http://www.rectoria.ucr.ac.cr/site/wpcontent/uploads/2014/09/1957.pdf

Universidad de Costa Rica (UCR). (2015). Historia de la Universidad de Costa Rica. Recuperado de www.ucr.ac.cr/acerca-u/historia-simbolos/historia.html

Universidad de Costa Rica (UCR). (2016). Carreras. Recuperado de http://www.ucr.ac.cr/estudiantes/carreras/

Vicerrectoría de Acción Social, Universidad de Costa Rica. (2016). ¿Qué es la Acción Social? Recuperado de http://accionsocial.ucr.ac.cr/accion-social

Vicerrectoría de Docencia, Universidad de Costa Rica. (2016a). Quiénes somos. Recuperado de http://vd.ucr.ac.cr/nosotros/

Vicerrectoría de Docencia, Universidad de Costa Rica. (2016b). Unidad de Apoyo a la Docencia Mediada con Tecnologías de la Información y Comunicación (METICS). Recuperado de http://vd.ucr.ac.cr/nosotros/metics/

Vicerrectoría de Docencia, Universidad de Costa Rica. (2016c). Red Institucional de Formación y Evaluación Docente (RIFED). Recuperado de http://vd.ucr.ac.cr/nosotros/red-institucional-de-formacion-y-evaluacion-docente/

Vicerrectoría de Docencia, Universidad de Costa Rica. (2016d). Cátedras Internacionales. Recuperado de http://vd.ucr.ac.cr/index.php/catedras-internacionales

Vicerrectoría de Docencia, Universidad de Costa Rica. (2016e). Docencia multiversa. Recuperado de http://vd.ucr.ac.cr/index.php/docencia-multiversa

Vicerrectoría de Docencia, Universidad de Costa Rica. (2016f). Proyectos de Docencia. Recuperado de http://vd.ucr.ac.cr/index.php/proyectos

Vicerrectoría de Investigación, Universidad de Costa Rica. (2016). Información general. Recuperado de http://www.vinv.ucr.ac.cr/informacion-general-de-la-vicerrectoria-deinvestigacion

Ysunza B., Marisa. (2010). Perfil de egreso y formación profesional: una estrategia metodológica en el diseño curricular. México: IISUE - Instituto de Investigaciones sobre la Universidad y la Educación de la Universidad Nacional Autónoma de México. 